Диагностика кардиотоксических нарушений ритма сердца и проводимости у онкологических больных с применением
телеметрической электрокардиографии

\author{
Н.П. Лямина ${ }^{\varpi 1}$, И.В. Погонченкова', С.В. Лямина \\ 'ГАУЗ «Московский научно-практический центр медицинской реабилитации, восстановительной \\ и спортивной медицины» Департамента здравоохранения г. Москвы, Москва, Россия; \\ ${ }^{2}$ ФГБОУ ВО «Московский государственный медицинский стоматологический университет \\ им. А.И. Евдокимова» Минздрава России, Москва, Россия \\ \lyana_n@mail.ru
}

\title{
Аннотация
}

цель. Оценка возможностей применения ЭКГ-телеметрии с целью повышения эффективности диагностики кардиотоксического эффекта у онкологических пациентов, получающих химиотерапию.

материал и методы. Дистанционное динамическое наблюдение в течение 6 мес проводилось у 31 кардиоонкологического пациента: у 17 пациентов онкологический диагноз - рак предстательной железы (РПЖ) и у 14 пациенток - рак молочной железы (РМж), средний возраст 53,6 (50,1; 56,8) года и 48,3 $(38,8 ; 57,1)$ года соответственно; функциональный класс сердечной недостаточности NYHA I-III; фракция выброса левого желудочка 47,4\% $(40,2 ; 57,3)$ и 43,2\% $(39,7 ; 58,9)$ соответственно; краткая шкала оценки псхического статуса (MMSE) - 27,6 $(25,4 ; 29,1)$ баллов и 28,2 $(25,1 ; 28,8)$ баллов соответственно. ЭКГ-телеметрия выполнялась по принципу аутотрансляции с использованием индивидуальных регистраторов электрокардиограммы (ЭКГ), мобильных устройств и интернет-приложения ECG Dongle («Нордавинд-Дубна», Россия). Для хранения, анализа информации и телемедицинского консультирования использована асинхронная телекардиологическая платформа CardioCloud. Условия регистрации ЭКГ: с частотой не менее 5 раз в день и дополнительно неоднократно при наличии симптоматики и желания пациента, продолжительность 10-20 мин.

Pезультаты. За 6 мес наблюдения у пациентов были дистанционно зарегистрированы ранее не документированные ЭКГ-изменения: у 87,0\% пациентов - синусовая тахикардия, на фоне которой регистрировались желудочковая экстрасистолия различных градаций (32,3\%), наджелудочковая экстрасистолия (22,6\%), преходящие тахизависимые блокады ножек пучка Гиса (9,7\%). Нарушения ритма в сочетании с депрессией сегмента $S T$ регистрировались в $64,7 \%$ случаев у пациентов с РПЖ и у 42,8\% с РМЖ. Пароксизмы фибрилляции предсердий были зарегистрированы у 17,6\% пациентов с РПЖ и у 14,3\% с РМЖ. У 35,5\% пациентов выявленные ЭКГ-изменения были бессимптомными.

Заключение. Удаленное динамическое наблюдение пациентов с онкологическими заболеваниями, получающих химиотерапию, со схемой регистрации ЭКГ ( $\geqslant 5$ раз в день, 10-20 мин) с применением ЭКГ-телеметрии является эффективным и недорогим методом диагностики ЭКГ-изменений при феномене кардиотоксичности. ключевые слова: ЭКГ-телеметрия, кардиоонкология, нарушения ритма сердца и проводимости.

Для цитирования: Лямина Н.П., Погонченкова И.В., Лямина С.В. Диагностика кардиотоксических нарушений ритма сердца и проводимости у онкологических больных с применением телеметрической электрокардиографии. СardioСоматика. 2020; 11 (2): 14-18. DOI: 10.26442/22217185.2020.2.200229

\section{Telemetric electrocardiography in diagnostics of cardiotoxic heart rhythm disorders and conductivity in oncologic patients}

\author{
Nadezhda P. Lyamina ${ }^{\bowtie I}$, Irena V. Pogonchenkova', Svetlana V. Lyamina² \\ 'Moscow Centre for Research and Practice in Medical Rehabilitation, Restorative and Sports Medicine, Moscow, \\ Russia; \\ ${ }^{2}$ Yevdokimov Moscow State University of Medicine and Dentistry, Moscow, Russia \\ 凶lyana_n@mail.ru
}

\begin{abstract}
Aim. Assessment of the possibility to use ECG-telemetry in order to increase the efficiency of cardiotoxic effect diagnosis in cancer patients with chemotherapy.

Material and methods. Remote dynamic monitoring for 6 months was carried out in 31 cardio-oncological patients (in 17 prostate cancer (PC), in 14 - breast cancer (BC), mean age was $53.6(50.1 ; 56.8)$ years and $48.3(38.8 ; 57.1)$ years, respectively; FC NYHA I-III; left ventricular ejection fraction 47.4\% (40.2; 57.3) and 43.2\% (39.7; 58.9), respectively; Mini-Mental State Examination from 30 to 24 points $-27.6(25.4 ; 29,1)$ and $28.2(25.1 ; 28.8)$, respectively. ECG-telemetry was performed according to the principle of auto-translation using individual ECG recorders, mobile devices and the ECG Dongle Internet application (Nordavind-Dubna, Russia). Storage, analysis of information and telemedicine consultation was perfomed with the CardioCloud asynchronous tele-cardiological platform. ECG was registered with a frequency of at least 5 times/day and additionally repeatedly in the presence of symptoms and patient desires, duration 10-20 minutes.
\end{abstract}


Results. Previously undocumented ECG changes were remotely recorded in patients for 6 months of follow-up: in 87.0\% of patients - sinus tachycardia, against which ventricular extrasystole of various gradations (32.3\%), supraventricular extrasystole (22.6\%), transient tachy-dependent blockade of the bundle of His legs were recorded (9.7\%). In 64.7\% of PC patients and in $42.8 \%$ of BC patients rhythm disturbances were detected in combination with depression of the ST segment. Paroxysms of atrial fibrillation were reported in $17.6 \%$ of PC patients and in $14.3 \%$ BC patients. In $35.5 \%$ of patients the detected ECG changes were asymptomatic.

Conclusion. Remote dynamic monitoring in cancer patients receiving chemotherapy involving ECG recording scheme $\geqslant 5$ times/day, 10-20 min) with ECG telemetry is an effective and unexpensive method for detecting ECG changes in the phenomenon of cardiotoxicity.

Key words: ECG-telemetry, cardio-oncology, cardiac rhythm and conductibility disorders.

For citation: Lyamina N.P., Pogonchenkova I.V., Lyamina S.V. Telemetric electrocardiography in diagnostics of cardiotoxic heart rhythm disorders and conductivity in oncologic patients. Cardiosomatics. 2020; 11 (2): 14-18. DOI: $10.26442 / 22217185.2020 .2 .200229$

\section{Введение}

Традиционно в развитых странах на первом месте среди причин смерти стоят заболевания сердца и сосудов, а на втором - онкологические заболевания [1]. В последние годы в онкологии произошел прорыв, благодаря ранней диагностике и более эффективному лечению улучшился прогноз и онкологические больные стали жить долыше [2]. Огромную роль в этом сыграла современная химиотерапия (XT). Но в результате неожиданно возникли и новые проблемы - кардиотоксичность ХТ и лучевой терапии, проводимой онкологическим больным [2-4]. Риск смерти от кардиологических причин у онкологических больных за последние годы вырос в 8 раз. По сути, получалось, что значительная часть из тех, кого вылечили от рака, умирали от сердечных проблем [5].

По данным крупного регистрового исследования, включающего данные 3234256 пациентов с онкологическими заболеваниями, наиболее высокий риск смерти от сердечно-сосудистых причин определен у пациентов, перенесших онкологическое заболевание следующих локализаций: мочевой пузырь $(19,4 \%)$, гортань $(17,3 \%)$, предстательная железа $(16,6 \%)$, тело матки $(15,6 \%)$, колоректальный рак $(13,7 \%)$, молочная железа (11,7\%). В наибольшей степени риск смерти от сердечно-сосудистых причин был выше у пациентов, у которых рак диагностирован до 35 лет и в первый год наблюдения [6].

Применение современных высокоэффективных схем ХТ онкологических заболеваний в 5-35\% случаев сопровождается кардиотоксическим эффектом, от бессимптомных изменений при электрокардиографии (ЭКГ) до развития острого коронарного синдрома и сердечной недостаточности, понижающих вероятность положительного результата терапии и ухудшающих жизненный прогноз пациента $[2,4,5,7]$. Одним из серьезных и не всегда своевременно выявляемых осложнений противоопухолевого лечения являются нарушения ритма сердца и проводимости. К сожалению, нарушения ритма сердца у части пациентов могут протекать бессимптомно. При этом многие нарушения ритма, такие как фибрилляция и трепетание предсердий, частая желудочковая экстрасистолия и особенно желудочковая тахикардия, могут приводить не только к серьезным гемодинамическим нарушениям, но и к внезапной смерти больного $[2,8]$.

Такой высокий риск сердечно-сосудистых заболеваний и сердечно-сосудистой смертности обусловлен как общими факторами риска, так и токсическими эффектами ХТ, повреждающим действием лучевой терапии [7]. Это определяет необходимость как в ранней диагностике, так и в ранней и агрессивной профилактике сердечно-сосудистых заболеваний у онкологических пациентов при постоянном и дли- тельном мониторинге за гемодинамическими показателями [2]. Оценка реальной распространенности кардиотоксичности часто затруднена и связана, в первую очередь, с коротким периодом наблюдения в рамках использования рутинных методов обследования пациентов и недостаточной скрининговой чувствительности традиционно применяемого метода ЭКГ. Использование специальных инструментальных и лабораторных инновационных методов исследования сердца позволяет выявлять более ранние, доклинические признаки поражения сердца.

Цель исследования - оценка возможностей применения ЭКГ-телеметрии с целью повышения эффективности диагностики кардиотоксического эффекта у онкологических пациентов, получающих ХТ.

\section{Материал и методы}

Исследование выполнялось как проспективное (продолжительность наблюдения - 6 мес). Письменное информированное согласие было получено от всех участников исследования.

Изучение эффективности дистанционного динамического наблюдения больных проводилось у 31 кардиоонкологического пациента, из которых у 17 пациентов онкологический диагноз - рак предстательной железы (РПЖ) и у 14 пациенток - рак молочной железы - РМЖ (табл. 1). Данные по кардиологическому диагнозу включенных в исследование представлены в табл. 1. Сроки включения в наблюдение были от 2 до 12 мес с момента начала ХТ; длительность ХТ в зависимости от стадии заболевания составила от 4 до 8 курсов. Продолжительность динамического наблюдения за пациентами составила 6 мес

Дополнительные условия включения: наличие у пациента или у членов его семьи мобильного устройства (смартфона, планшетного компьютера) с операционной системой Android не ниже 4.3 и выходом в Интернет. Критериями исключения являлись IV функциональный класс хронической сердечной недостаточности, наличие когнитивной дисфункции - количество баллов по Краткой шкале оценки психического статуса (Mini-Mental State Examination - MMSE) 23 и меньше, отсутствие навыков работы с мобильными устройствами. Оценка уровня когнитивных функций проводилась по шкале MMSE. Сумма 28-30 баллов оценивалась как отсутствие когнитивных нарушений; 24-27 баллов - легкие когнитивные нарушения [9].

Согласно протоколу исследования ЭКГ-телеметрия выполнялась по принципу аутотрансляции с использованием индивидуальных регистраторов ЭКГ, мобильных устройств и интернет-приложения ECG Dongle («Нордавинд-Дубна», Россия). Для хранения, анализа информации и телемедицинского консультирования использована асинхронная телекардиологическая платформа CardioCloud [10]. Условия регист- 


\begin{tabular}{|c|c|c|c|}
\hline \multirow{2}{*}{ Показатели } & \multicolumn{3}{|c|}{ Онкологический диагноз } \\
\hline & РПЖ (n=17) & PMЖ (n=14) & Статистическая значимость различий, $p$ \\
\hline Возраст, лет & $48-60$ & $32-60$ & \\
\hline Средний возраст, лет & $53,6(50,1 ; 56,8)$ & $48,3(38,8 ; 57,1)$ & $p<0,05$ \\
\hline \multicolumn{4}{|l|}{ Кардиологический диагноз, n (\%): } \\
\hline - артериальная гипертония & $12(70,6)$ & $9(64,4)$ & Недостоверно \\
\hline $\begin{array}{l}\text { - сердечная недостаточность } \\
\text { І-ІІІ Функционального класса }\end{array}$ & $17(100)$ & $8(57,1)$ & $p<0,05$ \\
\hline - нарушения ритма сердца & $2(11,8)$ & $1(7,1)$ & Недостоверно \\
\hline - нарушения проводимости & - & $1(7,1)$ & \\
\hline Значения по шкале MMSE, баллы & $27,6(25,4 ; 29,1)$ & $28,2(25,1 ; 28,8)$ & $p>0,05$ \\
\hline Фракция выброса левого жулудочка, \% & $47,4(40,2 ; 57,3)$ & $43,2(39,7 ; 58,9)$ & $p>0,05$ \\
\hline $\begin{array}{l}\text { Сроки включения в исследование } \\
\text { с момента начала XT, мес }\end{array}$ & $8,2(4,8 ; 10,6)$ & $5,4(2,7 ; 7,3)$ & $p<0,05$ \\
\hline
\end{tabular}

\begin{tabular}{|c|c|c|c|}
\hline Показатели & Bcero $(n=31)$ & PПЖ (n=17) & PMЖ (n=14) \\
\hline Синусовая тахикардия, n (\%), из них: & $27(87,0)$ & $15(88,2)$ & $12(85,7)$ \\
\hline • в сочетании с желудочковой экстрасистолией, n (\%) & $10(32,3)$ & $6(35,3)$ & $4(28,6)^{\star}$ \\
\hline • в сочетании с наджелудочковой экстрасистолией, n (\%) & $7(22,6)$ & $4(23,5)$ & $3(21,4)$ \\
\hline • преходящие тахизависимые блокады ножек пучка Гиса, n (\%) & $3(9,7)$ & $1(5,9)$ & $2(14,3)^{\star}$ \\
\hline Пароксизмы ФП, n (\%) & $5(16,1)$ & $3(17,6)$ & $2(14,3)$ \\
\hline Нарушения ритма в сочетании с депрессией сегмента ST, n (\%) & $17(54,8)$ & $11(64,7)$ & $6(42,8)^{*}$ \\
\hline Период ХT, мес & $12,8(9,7 ; 14,9)$ & $14,1(10,5 ; 16,8)$ & $11,0(8,6 ; 13,2)^{\star}$ \\
\hline
\end{tabular}

рации ЭКГ: с частотой не менее 5 раз в день и дополнительно неоднократно при наличии симптоматики и желания пациента; продолжительность 10-20 мин. Данные ЭКГ анализировались внешним врачом. Оценивались: частота сердечных сокращений средняя, наличие нарушений ритма и проводимости, динамика сегмента ST и зубцов; продолжительность $Q T$. Информация о физиологических параметрах доставлялась путем заполнения электронной формы «Дневник самоконтроля пациента». В дневнике фиксировались артериальное давление, частота сердечных сокращений, жалобы. Пациентам проводилась стандартная терапия, назначенная с учетом клинического статуса. При необходимости корректировалась лекарственная терапия.

Статистический анализ полученных результатов проводился с использованием программы Statistica 10 (StatSoft Inc., США). Проверка гипотезы нормального распределения осуществлялась с помощью теста Шапиро-Уилка. Количественные признаки представлены в виде медианы (Me) и квартилей (нижний квартиль; верхний квартиль). Для качественных показателей указывали n (\%), где n - абсолютное число, \% относительная величина в процентах. Статистически значимыми считались различия при $p<0,05$.

\section{Результаты}

При стандартном ЭКГ-исследовании (ЭКГ в 12 отведениях в течение 1 мин) у кардиоонкологических пациентов на этапе включения в исследование документированные ЭКГ-изменения были зарегистрированы только в виде нарушений ритма сердца (нечастая наджелудочковая и желудочковая экстрасистолия) и нарушений проводимости (атриовентрикулярная блокада 1-й степени) у 12,9\% пациентов: у 2 пациентов с РПЖ и у 2 с РМЖ (см. табл. 1).
За период 6 мес динамического наблюдения пациентов, продолжающих в этот период ХТ, дистанционно, с использованием индивидуальных регистраторов ЭКГ - ECG Dongle, были зарегистрированы ранее не документированные ЭКГ-изменения (табл. 2). В структуре ЭКГ-изменений значительно преобладала у 87,0\% пациентов синусовая тахикардия, на фоне которой регистрировались: желудочковая экстрасистолия различных градаций (32,3\%), наджелудочковая экстрасистолия $(22,6 \%)$, преходящие тахизависимые блокады ножек пучка Гиса (9,7\%).

Тяжесть и частота зарегистрированных эктопических нарушений ритма сердца как у пациентов с РПЖ, так и у пациенток с РМЖ была почти одинаковой.

Пароксизмы фибрилляции предсердий (ФП) были зарегистрированы как у пациентов с РПЖ, так и у пациенток с РМЖ: в 17,6 и 14,3\% соответственно, несмотря на более короткий период ХТ у пациенток с РМЖ - 11,0 мес против 14,1 мес $(p<0,05)$ у пациентов с РПЖ и более молодой возраст пациенток при РМЖ: средний возраст 48,3 года против 53,6 года $(p<0,05)$ соответственно.

Больше чем у $1 / 2$ пациентов $(54,8 \%)$ были выявлены сложные ЭКГ-изменения в форме сочетания синусовой тахикардии и наджелудочковых нарушений ритма (экстрасистолия, эпизоды ФП), синусовой тахикардии, желудочковой экстрасистолии и депрессии сегмента $S T$ (см. табл. 2). У пациентов с РПЖ нарушения ритма в сочетании с депрессией сегмента $S T$ выявлялись чаще, чем у пациенток с РМЖ: в 64,7 и 42,8\% соответственно. Эти различия могут быть определены как большей продолжительностью периода ХТ у пациентов с РПЖ - 14,1 $(10,5 ; 16,8)$ мес против $11,0(8,6 ; 13,2)$ мес у пациенток с РМЖ $(p<0,05)$, так и тяжестью кардиологического статуса (см. табл. 1). 
Выявленные ЭКГ-изменения в виде нарушений ритма сердца, проводимости и депрессии сегмента $S T$ у 64,5\% пациентов были симптомными, а в остальных случаях (35,5\%) пациенты жалоб на сердцебиение, перебои в работе сердца, стенокардитические боли не предъявляли.

Однако, по данным стандартного ЭКГ-исследовании (ЭКГ в 12 отведениях в течение 1 мин), проведенного по завершении 6-месячного динамического наблюдения за онкологическими пациентами, синусовая тахикардия регистрировалась в 3 раза реже только у 29,0\% (9 пациентов), нарушения ритма в сочетании с депрессией сегмента $S T$ регистрировалась в 2 раза реже - у 25,8\% (8 пациентов), а преходящие тахизависимые блокады ножек пучка Гиса и пароксизмы ФП при стандартном ЭКГ-исследовании не были зарегистрированы ни у одного пациента.

\section{Обсуждение}

В настоящее время во всем мире активно развивается направление кардиоонкологии, что обусловлено как ростом числа кардиоонкологических больных, так и отсутствием единого представления об основных профилактических, диагностических, терапевтических и реабилитационных подходов к проблеме [2]. Известно, что противоопухолевая терапия весьма токсична, многие химиопрепараты оказывают прямое повреждающее действие на клетки сердца и сосудов, что приводит к развитию тяжелых нарушений ритма сердца и проводимости, снижению его насосной функции, развитию дилатационной кардиомиопатии и сердечной недостаточности $[4,7,8]$. Нередко у пациентов с РМЖ и РПЖ используется комбинация химиотерапевтических препаратов, что кумулирует их кардиотоксическое действие $[4,5]$. Одним из серьезных и не всегда своевременно выявляемых осложнений противоопухолевого лечения являются нарушения ритма сердца и проводимости [8]. К сожалению, нарушения ритма сердца у ряда пациентов могут протекать бессимптомно. При этом многие нарушения ритма, такие как фибрилляция и трепетание предсердий, частая желудочковая экстрасистолия и особенно желудочковая тахикардия, могут приводить не только к серьезным гемодинамическим нарушениям, острым сосудистым событиям, но и к внезапной смерти больного $[4,5]$. Варианты нарушений ритма и проводимости могут варьировать и сочетаться между собой, приводить к тяжелой клинической симптоматике и быть угрожающими для жизни. Ухудшение состояния сердечно-сосудистой системы при проведении ХТ или таргетной терапии заставляет редуцировать дозировки препаратов, а иногда и полностью отказаться от продолжения әффективной противоопухолевой терапии $[2,4,5]$. В документе «Согласованное заключение экспертов ISHNE-HRS 2017 по амбулаторному мониторированию ЭКГ и наружному мониторированию деятельности сердца/телеметрии» определено, что использование современных устройств наружного кардиотелемониторинга в режиме реального времени - мобильной ЭКГ-телеметрии - позволяет своевременно диагностировать нарушения ритма и осуществлять беспроводную передачу данных практически в режиме реального времени, улучшая диагностическую значимость и существенно увеличивая эффективность при простоте использования [11].

Поэтому использование у онкопациентов мобильной ЭКГ-телеметрии в процессе проведения ХТ требуется определять онкопациентам индивидуально, с учетом симптоматики и получаемой терапии. При- менение систем наружного кардиотелемониторинга в режиме реального времени - мобильной ЭКГ-телеметрии - у онкобольных в процессе проведения ХТ и дальнейшем наблюдении за ними позволяет своевременно выявлять нарушения ритма сердца, проводимости, а также ЭКГ-изменения, характеризующие кардиотоксичность.

В группе обследуемых с РМЖ и РПЖ, получающих XT, за 6 мес дистанционного динамического наблюдения с использованием индивидуальных регистраторов ЭКГ - ECG Dongle были выявлены бессимптомные ЭКГ-изменения более чем у $1 / 3$ (35,5\%) пациентов. У 54,8\% пациентов были выявлены сложные ЭКГизменения в форме сочетания синусовой тахикардии и наджелудочковых нарушений ритма (экстрасистолия, эпизоды ФП), синусовой тахикардии, желудочковой экстрасистолии и депрессии сегмента $S T$, что потребовало не только своевременной коррекции терапии, как противоопухолевой, так и кардиопротективной, с использованием альтернативных схем лечения, но и дополнительного обследования. В ранее проведенных исследованиях других авторов без ЭКГ-телемониторинга при стандартном обследовании (ЭКГ в 12 отведениях в течение 1 мин) у онкобольных, получающих ХТ, различные аритмии были обнаружены в 16-36\% случаев [2, 8]. Использование в данном исследовании дистанционного ЭКГ-контроля (ЭКГ-телеметрии) в режиме онлайн в любое время суток с продолжительностью до 10 мин с частотой не менее 5 раз в сутки позволило выявить у 16,1\% онкопациентов пароксизмы ФП. Это определило для них дополнительную коррекцию антикоагулянтной терапии, так как при ФП стоит выбор стратегии контроля ритма/частоты, профилактики тромбоэмболии и эффективного предупреждения острых сосудистых событий и, прежде всего, инсульта. У онкологических пациентов с ФП оценка тромбоэмболических рисков (по шкале $\mathrm{CHA}_{2} \mathrm{DS}_{2}-\mathrm{VASc}$ ) и риска развития кровотечений (по шкале HAS-BLED) является сложной задачей, требующей индивидуального подхода с учетом симптомов пациента и проводимой терапии [2, 12], поэтому своевременная диагностика крайне важна.

В структуре ЭКГ-изменений у обследуемых пациентов значительно преобладала синусовая тахикардия у 87,0\%, на фоне которой, помимо желудочковой экстрасистолии и наджелудочковой экстрасистолии различных градаций, у каждого десятого пациента могут регистрироваться преходящие тахизависимые блокады ножек пучка Гиса. Нередко нарушения проводимости вследствие воздействия ХТ в дальнейшем становятся хроническими, могут приводить к тяжелой клинической симптоматике и быть угрожающими для жизни $[6,7,12]$. Поэтому для онкопациентов очень важна их ранняя диагностика, которая становится возможной для пациентов при использовании ЭКГ-телеметрии в режиме онлайн.

\section{Заключение}

Удаленное динамическое наблюдение пациентов с онкологическими заболеваниями: РПЖ и РМЖ, получающих ХT, со схемой регистрации ЭКГ ( $\geqslant 5$ раз в день, 10-20 мин) современными устройствами наружного кардиотелемониторинга в режиме реального времени - мобильной ЭКГ-телеметрии в сочетании с консультированием специалиста - является достаточно эффективным и недорогим методом диагностики феномена кардиотоксичности. Основным достоинством ЭКГ-телеметрии у онкологических пациентов являются возможность ранней диагностики 
кардиологических симптомов в виде нарушений ритма сердца, проводимости, депрессии сегмента $S T$, других ЭКГ-изменений и особенно бессимптомных кардиоваскулярных нарушений на протяжении всего периода XТ, а также своевременное и обоснованное назначение медикаментозной терапии.

Конфблим интересов. Авторы заявляют об отсутствии конфликта интересов.

Conflict of interests. The authors declare that there is not conflict of interests.

\section{Литература/References}

1. Bray F et al. Global Cancer Statistics 2018: GLOBOCAN Estimates of Incidence and Mortality Worldwide for 36 Cancers in 185 Countries. Cancer J Clin 2018; 10: 1-31. https://onlinelibrarydddd.wiley.com/doi/full/10.3322/caac.21492

2. Zamorano JL, Lancellotti P, Munoz DR et al; ESC Scientific Document Group. ESC Position Paper on cancer treatments and cardiovascular toxicity developed under the auspices of the ESC Committee for Practice Guidelines: The Task Force for cancer treatments and cardiovascular toxicity of the European Society of Cardiology (ESC). Eur HeartJ 2016; 37 (36): 2768-801.DOI: 10.1093/eurbeartj/ebw211

3. Soultati A, Mountzios G, Avgerinou C et al. Endothelial vascular toxicity from chemotherapeu tic agents: Preclinical evidence and clinical implications. Cancer Treatment Rev 2012; 38 (5): 473-83.https://doi.org/10.1016/j

4. Lancellotti P, Suter TM, Lopez-Fernandez T. Cardio-Oncology Services: rationale, organization, and implementation. Eur HeartJ 2019; 40: 1756-63.

5. Snipelisky D, Park JY, Lerman A et al. How to develop a cardiooncology clinic. Heart Fail Clin 2017; 13: 347-59.

6. Kathleen M, Sturgeon A. Population-based study of cardiovascular disease mortality risk in US cancer patients. Eur HeartJ 2019; 25 (1-3): ebz766. https://doi.org/10.1093/eurheartj/ehz766
7. Floyd JD, Nguyen DT, Lobins RL et al. Cardiotoxicity of cancer therapy. J Clin Oncol 2005; (23): 7685-96. DOI: 10.1200/ JCO.2005.08.78928

8. Челобитько ЕГ, Королев С.В., Конов А.В.и др. Тахиаритмии у пациентов с онкологическими заболеваниями. Клиническая практика. 2017; 4: 76-88.

[Chelobit'ko E.G., Korolev S.V., Konov A.V. et al. Takhiaritmii u patsientov s onkologicheskimi zabolevaniiami. Klinicheskaia praktika.2017; 4: 76-88 (in Russian).]

9. Folstein MF, Folstein SE, McHugh PR. «Mini-mental state $\gg$. A practical method for grading the cognitive state of patients for the clinician. J Psychiatric Res 1975; 12 (3): 189-98. DOI: 10.1016/0022-3956(75)90026-6

10. Лямина Н.П., Котельникова Е.В. Медико-технологические аспекты реабилитационного консультирования с позииий «ллектронного» здравоохранения. Кардиоваскулярная терапия и профилактика. 2018; 5: 59-64.

[Liamina N.P, Kotel'nikova E.V.Mediko-tekbnologicheskie aspekty reabilitatsionnogo konsul'tirovaniia s pozitsii "elektronnogo' zdravookhraneniia. Kardiovaskuliarnaia terapiia i profilaktika. 2018; 5: 59-64 (in Russian).]

11. Steinberg J.S., Varma N., Cygankiewicz I. и др. Согласованное заключение экспертов ISHNE-HRS 2017 по амбулаторному мониторированию ЭКГ и наружному мониторированию деятельности сердиа/телеметрии. Кардиология. 2018; 1: $16-64$.

[Steinberg J.S., Varma N., Cygankiewicz I. et al. Coglasovannoe zakliuchenie ekspertov ISHNE-HRS 2017 po ambulatornomu monitorirovaniiu EKG i naruzhnomu monitorirovaniiu deiatel'nosti serdtsa/telemetrii. Kardiologiia. 2018; 1: 16-64 (in Russian).]

12. Yeh ET, Bickford CL. Cardiovascular complications of cancer therapy: incidence, pathogenesis, diagnosis, and management.J Am Coll Cardiol 2009; 53 (24): 2231-47. https://doi.org/ 10.1016/j.jacc.2009.02.050

\section{Информация об авторах / Information about the authors}

Лямина Надежда Павловна - д-р мед. наук, проф., зав. отд. медицинской реабилитации ГАУЗ МНПЦ МРВСМ. E-mail: Iyana_n@mail.ru

Погонченкова Ирэна Владимировна - акад. Академии медико-технических наук, д-р мед. наук, дир. ГАУЗ МНПЦ МРВСМ, гл. внештат. специалист по медицинской реабилитации и санаторно-курортному лечению Департамента здравоохранения г. Москвы

Лямина Светлана Владимировна - д-р мед. наук, проф. каф. патологической физиологии, проф. каф. пропедевтики внутренних болезней и гастроэнтерологии, ФГБОУ ВО «МГМСУ им. А.И. Евдокимова»
Nadezhda P. Lyamina - D. Sci. (Med.), Prof., Moscow Centre for Research and Practice in Medical Rehabilitation, Restorative and Sports Medicine. E-mail: Iyana_n@mail.ru

Irena V. Pogonchenkova - D. Sci. (Med.), Moscow Centre for Research and Practice in Medical Rehabilitation, Restorative and Sports Medicine

Svetlana V. Lyamina - D. Sci. (Med.), Prof., Yevdokimov Moscow State University of Medicine and Dentistry 